\title{
Budget impact of somatostatin analogs as treatment for metastatic gastroenteropancreatic neuroendocrine tumors in US hospitals
}

This article was published in the following Dove Press journal:

ClinicoEconomics and Outcomes Research

16 August 2017

Number of times this article has been viewed

\author{
Jesse D Ortendahl' \\ Sonia J Pulgar ${ }^{2}$ \\ Beloo Mirakhur ${ }^{3}$ \\ David Cox ${ }^{3}$ \\ Tanya GK Bentley' \\ Alexandria T Phan ${ }^{4}$ \\ 'Health Economics, Partnership \\ for Health, LLC, Beverly Hills, \\ CA, USA; ${ }^{2}$ Health Economics \\ and Outcomes Research, Ipsen \\ Biopharmaceuticals, Basking Ridge, NJ, \\ USA; ${ }^{3}$ Medical Affairs, Oncology, Ipsen \\ Biopharmaceuticals, Basking Ridge, NJ, \\ USA; ${ }^{4} \mathrm{GI}$ Medical Oncology, University \\ of New Mexico Comprehensive \\ Cancer Center, Albuquerque, NM, \\ USA
}

Correspondence: Jesse D Ortendah Partnership for Health Analytic Research, LLC, 280 S. Beverly Dr. \#404, Beverly Hills, CA, USA

Tel +I 3108589555

Fax + I 3108589552

Email jesse@pharllc.com
Objective: With the introduction of new therapies, hospitals have to plan spending limited resources in a cost-effective manner. To assist in identifying the optimal treatment for patients with locally advanced or metastatic gastroenteropancreatic neuroendocrine tumors, budget impact modeling was used to estimate the financial implications of adoption and diffusion of somatostatin analogs (SSAs).

Patients and methods: A hypothetical cohort of 500 gastroenteropancreatic neuroendocrine tumor patients was assessed in an economic model, with the proportion with metastatic disease treated with an SSA estimated using published data. Drug acquisition, preparation, and administration costs were based on national pricing databases and published literature. Octreotide dosing was based on published estimates of real-world data, whereas for lanreotide, real-world dosing was unavailable and we therefore used the highest indicated dosing. Alternative scenarios reflecting the proportion of patients receiving lanreotide or octreotide were considered to estimate the incremental budget impact to the hospital.

Results: In the base case, 313 of the initial 500 gastroenteropancreatic neuroendocrine tumor patients were treated with an SSA. The model-predicted per-patient cost was US $\$ 83,473$ for lanreotide and US $\$ 89,673$ for octreotide. With a hypothetical increase in lanreotide utilization from $5 \%$ to $30 \%$ of this population, the annual model-projected hospital costs decreased by US $\$ 488,615$. When varying the inputs in one-way sensitivity analyses, the results were most sensitive to changes in dosing assumptions.

Conclusion: Results suggest that factors beyond drug acquisition cost can influence the budget impact to a hospital. When considering preparation and administration time, and real-world dosing, use of lanreotide has the potential to reduce health care expenditures associated with metastatic gastroenteropancreatic neuroendocrine tumor treatments.

Keywords: health economics, oncology, model, costs, real-world evidence, lanreotide

\section{Introduction}

Gastroenteropancreatic neuroendocrine tumors (GEP-NETs) are rare neoplasms that originate from the secretory cells of the neuroendocrine system. ${ }^{1,2}$ Recent analyses have suggested that the incidence of neuroendocrine tumors (NETs) is increasing and that the prevalence of NETs in the USA may exceed 100,000. ${ }^{3,4}$ While there is no single treatment algorithm that is used universally, surgical procedures are typically performed for curative purposes. In patients for whom surgery is not possible, palliative options, including somatostatin analogs (SSAs), are used to reduce tumor burden in locally advanced and metastatic gastrointestinal neuroendocrine tumors and pancreatic neuroendocrine tumors. ${ }^{5}$ Lanreotide depot (Somatuline Depot), an SSA, is currently 
approved in the USA to improve progression-free survival (PFS) for adult patients with unresectable, well or moderately differentiated, locally advanced or metastatic GEP-NETs. Another SSA used in this patient population is octreotide long-acting release (Sandostatin LAR), which is US Food and Drug Administration approved to treat severe diarrhea/flushing episodes associated with metastatic carcinoid tumors. These agents were assessed in two double-blind, placebocontrolled trials: the PROMID study for octreotide $\mathrm{LAR}^{6}$ and the CLARINET trial for lanreotide depot. ${ }^{7}$ CLARINET was an international study across 14 countries that enrolled 204 patients with tumors originating from the midgut, as well as those with tumors originating in the pancreas and hindgut. PROMID was conducted in Germany and included 85 patients with NETs originating in the midgut and those with unknown tumor origin but suspected to be from the midgut. Both SSAs were assessed at their maximum recommended doses in the USA, $120 \mathrm{mg}$ lanreotide and $30 \mathrm{mg}$ octreotide injections, each administered once every 4 weeks. Both trials found statistically significant differences in the hazard ratio for progression or death for the SSAs compared to placebo. At the conclusion of the CLARINET trial, median PFS for patients treated with placebo was 18.0 months, while the median PFS for patients receiving lanreotide was not reached at 96 weeks. During the CLARINET open-label extension study, the median PFS for lanreotide was reached and estimated to be 32.8 months. The extension study also found fewer adverse events than during the core study for lanreotide patients, suggesting favorable long-term safety and tolerability. ${ }^{8}$ Those who survived through the conclusion of the PROMID study were further monitored, although treatment was left to the discretion of the local investigators and crossover was allowed. During this follow-up period, the overall survival was similar in patients receiving octreotide LAR (84.7 months) or placebo (83.7 months). ${ }^{9}$ Both lanreotide depot and octreotide LAR are included in the National Comprehensive Cancer Network treatment consensus guidelines and the National Comprehensive Cancer Network compendium. ${ }^{10,11}$ Therefore, a comparison of these two agents as treatments for GEP-NET patients is necessary despite differences in indications.

Increasing health care costs are well documented, and efforts to control costs are underway. ${ }^{12-14}$ In all disease areas, and especially in oncology, clinical decision makers face competing demands for limited resources and must determine methods for choosing optimal treatments for their patients. One consideration in this choice of pharmacologic interventions is evaluation of product acquisition and overall treatment costs. The focus on the cost of care goes beyond the costs incurred by health care payers, as a shift in payment landscape has led to changes where now providers and hospital systems also take on risk and are incentivized to control expenditures. Although the bottom line impact on a hospital system includes both the resources utilized and the corresponding reimbursement, these are often considered independently. In most scenarios, hospitals aim to decrease costs regardless of reimbursement environment, with the objective of increasing the efficiency and decreasing the overall costs to ensure optimal and successful delivery of care to patients.

Budget impact modeling is one method used to evaluate the cost of health care delivery. In such analyses, relevant costs for treating a given disease are incorporated into a mathematical model, and outcomes are assessed when varying allocation of patients across treatments. ${ }^{15,16}$ Budget impact models may consider factors that would impact the financial implications of treatment choice and predict health expenditures for a given disease over a finite period without considering explicit assumptions regarding the efficacy or safety beyond the financial impact of these important clinical outcomes. Results are beneficial in understanding how different factors impact overall costs and provide one piece of information to aid in allocating resources effectively and efficiently.

In treating metastatic GEP-NET (mGEP-NET) patients, the financial impact of treatment choice is unclear. There is significant variation in real-world dosing of SSAs, the injection method and success rate differ between treatments, and there are differences in preparation and administration time required for each therapy. This analysis of the overall costs of different SSAs will help institutional and hospital decision makers better identify potential sources of cost savings and efficiency.

\section{Patients and methods Overview}

An economic model was developed to estimate the budget impact of treating patients with mGEP-NETs using SSAs. The model compared two scenarios, the first scenario reflective of a hypothetical baseline utilization of lanreotide or octreotide and then an alternative scenario based on a hypothetical shift in utilization. Model inputs consisted of population data, relevant costs, and treatment utilization. The model assessed patients over a 1-year period, and outcomes included annual costs to a hospital system and the average cost per treated patient. The model schematic is shown in Figure 1.

\section{Model inputs}

The model included the following categories of inputs: population and epidemiologic estimates, product acquisition 


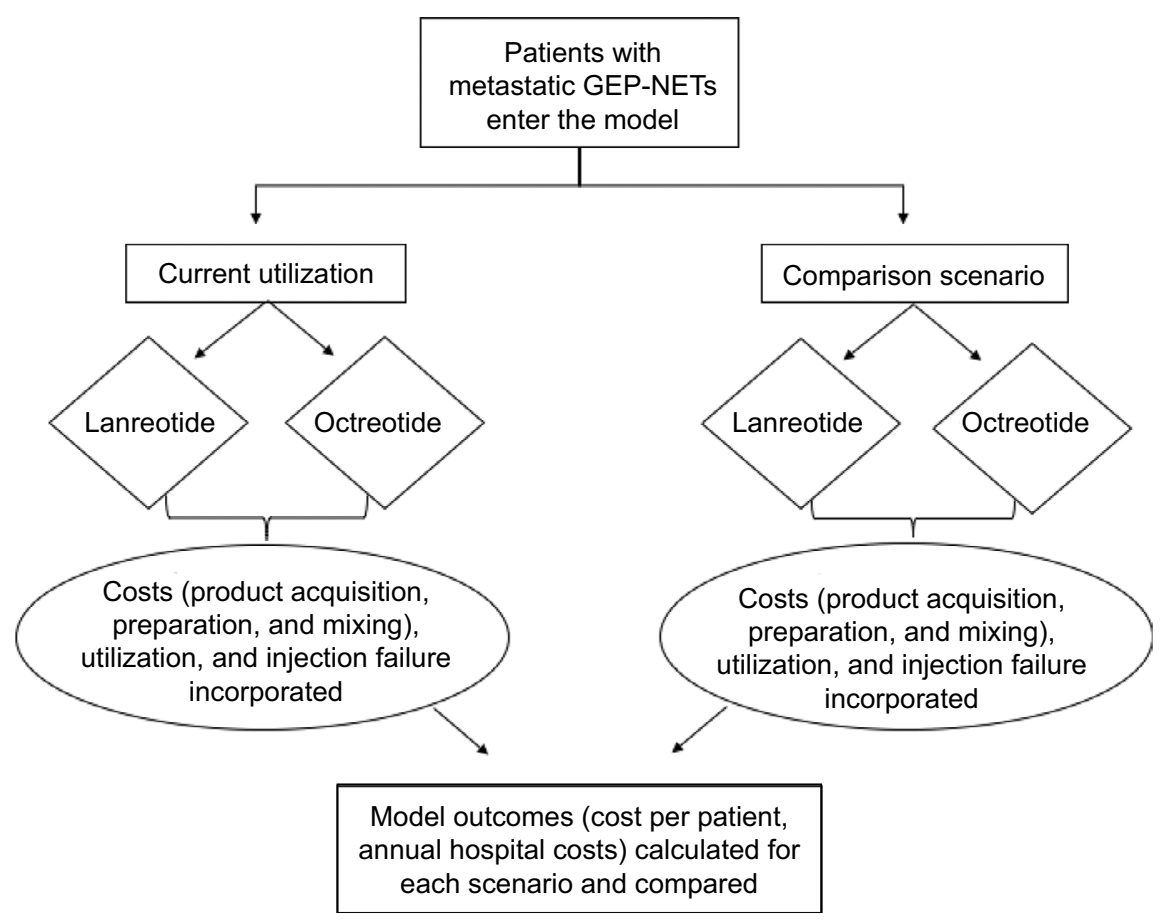

Figure I Model schematic.

Notes: The model schematic shows the flow of patients through the model for the two scenarios. In each scenario, those eligible for somatostatin analogs are treated with either lanreotide or octreotide. For each treatment, patients accrue costs based on the product acquisition and administration costs, which take into account dosing and the impact of failed injections. Total costs are estimated for each scenario, and compared to assess the costs of shifting utilization.

Abbreviation: GEP-NETs, gastroenteropancreatic neuroendocrine tumors.

and preparation/mixing costs, and product utilization. Inputs were based on published literature and pricing databases to the extent possible, and supplemented with market share data and expert opinion.

To estimate the financial impact for a hospital system or oncology department, we identified the expected number of patients eligible for treatment with an SSA at such facilities. To do so, we made an initial assumption that the facility would treat 500 GEP-NET patients annually. Of these, the patients included in the model were limited to those with metastatic disease and eligible for treatment with an SSA. A previously published US analysis found $80 \%$ of GEP-NET patients to be metastatic, a proportion similar to more recent estimates from outside the USA. ${ }^{17-20}$ Of these patients, it was assumed that $78 \%$ would be eligible for treatment with an SSA, based on a previously conducted US-based survey of physicians. ${ }^{21}$

Costs considered in the model included those related to product acquisition as well as preparation and mixing (Table 1). Product acquisition costs were calculated based on wholesale acquisition costs as of January $2017 .{ }^{22}$ Lanreotide depot is available in single-use, prefilled syringes of 60,90 , and $120 \mathrm{mg}$, and octreotide LAR is sold as single-use kits of 10,20 , and $30 \mathrm{mg}$ that require reconstitution. ${ }^{22-24}$ Multiple sources suggest that many patients receive above-indication
Table I Model population and cost parameters

\begin{tabular}{lll}
\hline Parameter & Value & Reference \\
\hline $\begin{array}{l}\text { Population } \\
\text { Facility size (\# GEP-NET patients) }\end{array}$ & 500 & Assumption \\
$\begin{array}{l}\text { Proportion of GEP-NET patients with } \\
\text { metastatic disease }\end{array}$ & $80.0 \%$ & {$[17]$} \\
$\begin{array}{l}\text { Proportion of metastatic GEP-NET } \\
\text { patients eligible for SSA }\end{array}$ & $78.2 \%$ & {$[21]$} \\
Costs $^{\mathrm{a}}$ & & \\
Lanreotide acquisition costs, mg & & \\
60 & US $\$ 4,087$ & [22] \\
90 & US $\$ 5,443$ & \\
120 & US $\$ 6,421$ & \\
Octreotide acquisition costs, mg & & \\
10 & US $\$ 2,83$ I & [22] \\
20 & US $\$ 3,709$ & \\
30 & US $\$ 5,554$ & \\
Preparation and mixing cost (per injection) & & \\
Lanreotide & US $\$ 0.83$ & [3I, 32] \\
Octreotide & US $\$ 4.12$ & \\
\hline
\end{tabular}

Note: aAcquisition costs based on WAC as of 01/24/2017.

Abbreviations: GEP-NET, gastroenteropancreatic neuroendocrine tumor; SSA, somatostatin analog; WAC, wholesale acquisition costs.

dosing of octreotide, and this was incorporated as a base case assumption in the model..$^{20,25-30}$ Because the maximum package size is $30 \mathrm{mg}$, we assumed that patients receiving above maximum doses were administered a single injection 
of two $20 \mathrm{mg}$ kits (for a $40 \mathrm{mg}$ injection) or a single injection using two $30 \mathrm{mg}$ kits (for a $60 \mathrm{mg}$ injection). To incorporate the time cost of preparing, mixing, and administering each product, we used publicly available wage rates and multiplied them by a published observational study of time spent preparing each product. It was assumed that a nurse practitioner would be preparing and administering injections, and we therefore used a wage rate from May 2014 for such a nurse in a specialty hospital. ${ }^{31}$ Time estimates were 329 seconds per injection for octreotide and 66 seconds for lanreotide, with differences due to additional efforts for octreotide because it requires reconstitution. ${ }^{32}$

To estimate the total costs for the hospital system, the costs per treated patient were multiplied by the number of patients receiving each dose for both products across the two scenarios. In the first scenario, $5 \%$ of patients received lanreotide and $95 \%$ of patients received octreotide, based on an unpublished claims analysis of US patients from 2008 to 2010. An alternative scenario assumed a hypothetical shift in utilization, leading to $30 \%$ of patients receiving lanreotide and $70 \%$ receiving octreotide. In both scenarios, the same amount of each product and frequency of injection was used. For lanreotide, currently, there are no studies in the USA reporting utilization beyond the maximum indicated dose of $120 \mathrm{mg}$ every 4 weeks; therefore, it was assumed all patients were treated in this manner. For octreotide, while the maximum indicated dose is $30 \mathrm{mg}$ every 4 weeks, multiple sources report a significant portion of patients receiving either higher doses or more frequent injections. ${ }^{20,25-30}$ Based on a previously published assessment of seven National Comprehensive Cancer Network treatment centers, it was assumed that $64 \%$ of patients received the maximum dose of $30 \mathrm{mg}$ every 4 weeks, while $36 \%$ of patients received octreotide either more frequently or at a higher dose. ${ }^{30}$ Specifically, $18 \%$ were treated with octreotide at either 40 or $60 \mathrm{mg}$ every 4 weeks and $18 \%$ received injections once every 3 weeks or once every 2 weeks. ${ }^{30}$ Product utilization parameters are shown in Table 2.

\section{Analyses}

In the base case, we assessed the costs of treating patients with either SSA treatment. These results were presented in terms of the total cost per treated patient, and divided between those related to product acquisition and those related to preparation and mixing. We also calculated the financial implications of a shift in utilization of products between the initial scenario and the alternative scenario, to show the incremental cost of an increase in lanreotide utilization. In sensitivity analyses, key parameters were varied to assess the impact on model
Table 2 Product utilization parameters

\begin{tabular}{|c|c|c|}
\hline Parameter & Value & Reference \\
\hline \multicolumn{3}{|l|}{ Utilization by product } \\
\hline \multicolumn{3}{|l|}{ Initial scenario } \\
\hline Lanreotide & $5 \%$ & [33] \\
\hline Octreotide & $95 \%$ & \\
\hline \multicolumn{3}{|l|}{ Comparison scenario } \\
\hline Lanreotide & $30 \%$ & Assumption \\
\hline Octreotide & $70 \%$ & \\
\hline \multicolumn{3}{|l|}{ Dosing by product ${ }^{\mathrm{a}}$} \\
\hline \multicolumn{3}{|l|}{ Lanreotide } \\
\hline 60-90 mg I $\times$ every 4 weeks & $0 \%$ & {$[24]$} \\
\hline $120 \mathrm{mg}$ I $\times$ every 4 weeks & $100 \%$ & \\
\hline \multicolumn{3}{|l|}{ Octreotide } \\
\hline I0-20 mg I $\times$ every 4 weeks & $0 \%$ & [30] \\
\hline $30 \mathrm{mg} \mathrm{I} \times$ every 4 weeks & $64 \%$ & \\
\hline$>30 \mathrm{mg} \mathrm{I} \times$ every 4 weeks $^{\mathrm{b}}$ & $18 \%$ & \\
\hline $30 \mathrm{mg}>1 \times$ every 4 weeks $^{c}$ & $10 \%$ & \\
\hline$>30 \mathrm{mg}>1 \times$ every 4 weeks $^{\mathrm{d}}$ & $8 \%$ & \\
\hline
\end{tabular}

Notes: a Dosing equivalent across scenarios. ${ }^{\mathrm{b}} \mathrm{Among}$ patients receiving $>30 \mathrm{mg}$ injections, I $4 \%$ received $40 \mathrm{mg}$ delivered as two $20 \mathrm{mg}$ injections and $4 \%$ received $60 \mathrm{mg}$ administered as two $30 \mathrm{mg}$ injections. 'Among patients receiving octreotide more frequently than once every 4 weeks, $3 \%$ received injections once every 3 weeks and $7 \%$ received injections every 2 weeks. ${ }^{\mathrm{d}}$ Among patients receiving $>30 \mathrm{mg}$ more frequently than once every 4 weeks, $6 \%$ received $40 \mathrm{mg}$ every 3 weeks and $2 \%$ received $60 \mathrm{mg}$ every 3 weeks.

outcomes. Additionally, a scenario analysis was conducted to explore the impact of injection failure. In the base case, it was assumed there was no financial impact of injection failure. However, in this scenario, we assumed that $25 \%$ of octreotide injections did not reach the intramuscular level, based on findings from a quality improvement study conducted at MD Anderson with trained health care providers, ${ }^{33}$ and in those cases, we assumed a repeat injection was needed.

\section{Results}

\section{Base case}

In the base case analysis, the model predicted that each patient treated with lanreotide would accrue an annual cost of US\$83,473, compared to US\$89,673 for octreotide. The current practice patterns, which indicate a mix of $5 \%$ of patients receiving lanreotide and $95 \%$ of patients receiving octreotide, would result in annual costs of US\$27,970,455 for a hospital with 500 GEP-NET patients. With a shift in utilization leading to $30 \%$ of patients receiving lanreotide and $70 \%$ receiving octreotide, this cost decreased to US\$27,481,840, resulting in cost savings of US $\$ 488,615$. While the magnitude of cost savings will depend on the proportion of patients shifting treatment, there was a linear relationship between increasing lanreotide utilization and reducing costs. Cost savings for lanreotide were driven both by the difference in product acquisition costs between products at commonly used doses 
as well as the reduction in preparation and mixing costs with lanreotide. Base case results are presented in Table 3.

\section{Sensitivity analyses}

In one-way sensitivity analyses examining uncertainty in each parameter individually, we found that the results were most sensitive to assumptions related to proportion of patients receiving above-indication dosing of octreotide and the acquisition costs of the two products. When it was assumed that all patients on octreotide received a single $30 \mathrm{mg}$ injection once every 4 weeks, the model predicted that a shift in utilization would result in an increase in costs of US\$878,489. If the proportion of octreotide patients with above-indicated dosing increased from the base case value of $36 \%-50 \%$, the decrease in costs with additional lanreotide treatment was US $\$ 1,030,874$. When the proportion of octreotide patients receiving above-indicated dosing was $23 \%$, the two scenarios were roughly equivalent. When varying the acquisition costs of lanreotide $\pm 20 \%$, the budgetary impact with a shift in utilization ranged from a savings of US\$1,794,133 to a cost increase of US $\$ 816,903$, respectively. A similar change in octreotide pricing led to incremental costs between the current and comparator scenarios of an increase in costs of US $\$ 913,868$ to a savings of US $\$ 1,891,098$. Other parameters, such as the preparation and mixing costs, had a minimal impact on the results. The model inputs with the largest impact on model results are shown in the tornado diagram (Figure 2).

\section{Scenario analysis}

When considering the possibility of intramuscular injection failure, as is possible with octreotide but not lanreotide which is administered via deep subcutaneous injection, we found that at $25 \%$ failure and assuming a repeat injection upon failure, the cost saving with a shift in utilization was US $\$ 2,242,876$. If the failure rate was half of the published value, or $12.5 \%$, the savings with increased lanreotide utilization was US $\$ 1,365,746$ for the hospital, or US $\$ 17,465$ per treated patient. ${ }^{33}$

\section{Discussion}

In this model-based analysis, we found that increasing utilization of lanreotide would result in cost savings to the hospital system. While the base case result of US $\$ 488,615$ in savings was based on a specific shift from $5 \%$ to $30 \%$ utilization of lanreotide within a hospital with 500 GEP-NET patients, the more general finding was that any shift toward higher lanreotide use would reduce expenditures. When comparing agents at the highest indicated dose, lanreotide is more

Table 3 Base case resultsa,

\begin{tabular}{|c|c|c|c|c|c|}
\hline & \multicolumn{2}{|c|}{ Annual hospital costs } & \multicolumn{3}{|c|}{ Cost per treated patient } \\
\hline & Initial scenario & Comparison scenario & Product acquisition & Preparation and mixing & Total \\
\hline Lanreotide & US\$1,305,686 & US\$7,834,II5 & US\$83,473 & US\$II & US\$83,484 \\
\hline Octreotide & US\$26,664,769 & US\$19,647,725 & US\$89,673 & US\$59 & US $\$ 89,732$ \\
\hline Total & US\$27,970,455 & US $\$ 27,481,840$ & - & - & - \\
\hline Difference $^{c}$ & - & -US\$488,615 & - & - & US\$6,248 \\
\hline
\end{tabular}

Notes: a Current utilization defined as market share today, and comparison scenario defined as a hypothetical change in market share. ${ }^{b}$ Costs include medications, administration, and mixing. 'Difference reflects the change in total costs between the baseline and the comparator year. A negative number denotes a cost savings in comparator year.

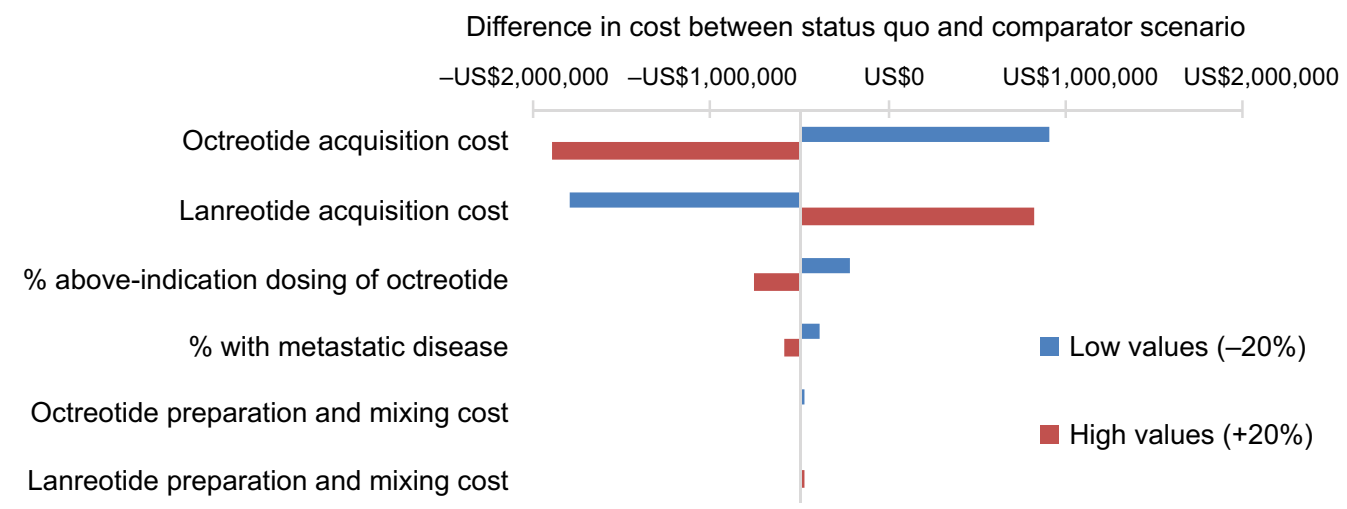

Figure 2 Sensitivity analysis results: tornado diagram.

Notes: The sensitivity analysis tornado diagram indicates the impact of parameter uncertainty on the model outcomes. Each horizontal bar shows the range of differences in costs of the comparator scenario compared with the status quo scenario when varying a given parameter by $\pm 20 \%$. As depicted, the parameter with the greatest impact on model results was product acquisition costs, followed by the proportion of patients getting above-indicated dosing of octreotide. 
expensive than octreotide. However, these results demonstrate the financial impact of real-world utilization that would be expected in clinical practice. These insights are especially relevant, given the increases in GEP-NET prevalence due to higher diagnoses rates and relatively long survival.

One area in which further research could be beneficial would be the implications of failed intramuscular injections. A key difference between the two products is that lanreotide is injected subcutaneously, whereas octreotide requires gluteal intramuscular injection. A previous analysis has shown, within a controlled study setting, that quality improvement initiatives can improve the success rate of intramuscular injections from $52 \%$ to $75 \%{ }^{33}$ However, even after such a training program, a quarter of intramuscular injections did not result in successful delivery of medication. The finding that intramuscular injections frequently do not reach the muscular level has also been reported across other disease areas, especially in obese patients. ${ }^{34-37}$ This issue is of increasing importance as obesity rates in the USA rise. The real-world impact of these failed doses is not completely understood, but it is hypothesized that they could lead to either more frequent or higher dose injections, repeat injections at a later time point, or a decrease in efficacy if the failed injection was not identified; all of these consequences have the potential to pose an incremental budget impact. In sensitivity analyses, we assessed the impact of the failed injections resulting in repeated injections, either at each instance or in $50 \%$ of cases. In both of these scenarios, the cost implications were significant and resulted in higher cost savings with lanreotide use.

Another factor that was found in the model to be influential was the dosing of each product in the real world. Currently, there is a lack of evidence from the USA of lanreotide being used above indication in mGEP-NET patients, though further investigation would help confirm this assumption. Outside the USA, there is some limited evidence of lanreotide being used more frequently than every 4 weeks; however, the implications are unclear, given that the treatment practices can differ greatly between settings. A survey of 110 Italian oncologists reported that lanreotide was primarily used at a maximum of $120 \mathrm{mg}$; however, $43 \%$ recalled using it more frequently than once every 4 weeks. However, the same survey found that $63 \%$ used octreotide more frequently than once every 4 weeks. ${ }^{38}$ A retrospective study of 118 Italian patients reported that $18 \%$ of patients received high-frequency SSA, with 15 of these patients treated with octreotide and six receiving lanreotide, but the proportions among the original 118 patients were not reported. ${ }^{39}$ An observational study of 273 patients across eight European countries who received lanreotide reported that $98 \%$ received doses between 60 and $120 \mathrm{mg}$ and $93 \%$ received injections every 4 weeks. The dosing for the small proportion not following US-indicated dosing was not described. ${ }^{40}$ Additionally, a case study from Belgium reported on a single patient who received lanreotide every 2 weeks. ${ }^{41}$ For octreotide, many studies report patients receiving either more frequent or higher dose injections. ${ }^{20,21,25-30}$ Whether this is to compensate for failed injections or to maintain the desired level of efficacy in treatment-experienced patients is unclear; however, to gain a full understanding of the economic implications of product use, this could be explored and analyzed further. Additionally, a recent Phase III trial of a new SSA considered octreotide $60 \mathrm{mg}$ every 4 weeks, or twice the indicated dose, suggesting those researchers felt that $60 \mathrm{mg}$ of octreotide was an appropriate dose to consider in practice. ${ }^{42}$ It is also important to note that there is no data to suggest that lanreotide depot $120 \mathrm{mg}$ is equivalent to 30 $\mathrm{mg}$ of octreotide LAR. Octreotide LAR is approved in some countries at a higher dose $(40 \mathrm{mg}){ }^{43}$

As an additional differentiating factor between treatments, the efforts required to prepare and administer octreotide exceed those for lanreotide. While lanreotide is packaged as individual, prefilled syringes, octreotide preparation involves reconstituting the product, waiting for it to be ready, and administering within a set amount of time. This increase in the amount of active provider time necessary to treat a single patient has been incorporated into the analysis based on insights from a controlled setting, ${ }^{32}$ but no time and motion study has been conducted in the real-world setting to identify the true time demands in clinical practice. The time costs may be underestimated, as preparation of octreotide also requires up to an hour of waiting between being mixed and being injected, whereas it is recommended that lanreotide should be left at room temperature for 30 minutes, neither of which are included in preparation time estimates used..$^{23,24}$ In the current context of care delivery, there is a nursing shortage and competing demands for those responsible for providing care. While it is unclear as to the extent clinical practice is prioritizing therapies that reduce provider time, it would be a method of increasing hospital throughput and/or reducing the burden on nurses.

This analysis should be considered as one source of information among other similar studies. A cost-minimization study presented at North American Neuroendocrine Tumor Society (NANETS) 2014 compared lanreotide and octreotide in patients with mGEP-NETs. ${ }^{44}$ Typically, in cost-effectiveness analyses, clinical data will be based on head-to-head clinical trials or indirect treatment comparisons will be conducted, to ensure a fair comparison is being made. 
In that analysis, the authors used results from the ELECT study, ${ }^{45}$ which only investigated the efficacy of lanreotide, to reflect symptom control for both agents, while the PROMID study, ${ }^{6,9}$ which only investigated octreotide, was used for the antitumor efficacy of both products. In that analysis, the two products were assumed to have equivalent efficacy without the rigor of a head-to-head study or a formal treatment comparison using published data, and it essentially was a cost-minimization analysis when comparing products at indicated doses. With these assumptions, this prior analysis found that use of lanreotide increased costs over a 3-year period and for the lifetime of patients. Another key difference between that study and the model described in this manuscript is the assumptions around utilization, including real-world dosing of SSAs. While the NANETS presentation assumed all patients received indicated doses of octreotide, we incorporated real-world utilization data to better reflect the expenditures expected in the USA.

Although we followed best practices in budget impact modeling and carefully selected the data to include, results should be considered in light of the limitations. Due to a lack of published data on real-world lanreotide utilization, we assumed that all patients receive the maximum indicated dose. While it is possible that some patients may receive above-indicated doses of lanreotide, there have not been published data indicating real-world use of $>120 \mathrm{mg}$ in the USA. As mentioned previously, there is uncertainty on the downstream economic implications of intramuscular injection failure. In the base case analysis, we did not consider the possibility of unsuccessful octreotide injections; however, this was explored in scenario analyses and found to influence costs. As was the case with the previous analysis from NANETS comparing the cost-effectiveness of these two products, the lack of head-to-head trials comparing efficacy measures prevented consideration of clinical benefits differing between treatments. Additionally, differences in patient populations between the PROMID ${ }^{6,9}$ and CLARINET $^{8}$ trials prohibited indirect comparisons. The lack of data on comparative effectiveness prevented consideration of differential costs based on treatment response, or costs that could be accrued following SSA discontinuation. Without evidence of clinical superiority, the choice of treatments becomes primarily based on costs. We conducted the analysis from the hospital perspective, such that indirect costs borne by the patient, such as time, transportation, and productivity losses, were not considered. We did not consider the differences between subgroups of mGEP-NET patients, for example, by functional status or the site of tumor origin; however, this would be an area for further investigation in the future. If such research indicates that there are patients who can be identified a priori as being more likely to benefit from a specific therapy, this method of practicing personalized medicine should be undertaken. We only considered the branded versions of these therapies, and if biologic equivalents become available and widely used, the financial impact should be reassessed. Additionally, varying the acquisition costs of each product was found to have a large impact on model results. As pricing negotiations can shift the drug costs to hospitals, the results of sensitivity analyses should be considered in cases where facilities face lower costs, and negotiations related to drug pricing would be a method of reducing overall treatment costs. Both products can also be used for other indications, for example, acromegaly; however, this study only examined the cost consequences of treatment choice for GEP-NET patients.

\section{Conclusion}

In oncology treatment, there is increasing concern over the cost of providing care while striving to get the most cost-effective and disease controlling regimen to the patient. Because of this impetus to consider the value of products and allocate the resources efficiently, different groups including American Society of Clinical Oncology have been developing tools to help compare products and assess new technologies. These types of budget impact analyses can help provide insights into the full cost of treatment choice, beyond a simple comparison of acquisition costs at the indicated dosing. There is a lack of published evidence on the expected resource utilization in the real world with mGEP-NET treatments. The finding that increased utilization of lanreotide can reduce costs could be beneficial to payers, hospital systems, clinicians, and others concerned about the cost of care for mGEP-NET patients.

\section{Acknowledgment}

This study was funded by Ipsen Biopharmaceuticals. No assistance in the preparation of this article is to be declared.

\section{Authors' contributions}

JDO, TGKB, SJP, and DC were involved in the conception and design of the study. JDO, TGKB, SJP, and ATP were involved in analysis and interpretation of the data. JDO, TGKB, SJP, BM, DC, and ATP were involved in drafting the paper, revising it critically for intellectual content, and providing final approval. All authors contributed toward data analysis, drafting and revising the paper and agree to be accountable for all aspects of the work. Authorship order was determined based on the level of contributions, with the exception of ATP who was selected as the last author to reflect the overarching clinical contributions to the work. 


\section{Disclosure}

JDO and TGKB are employees of Partnership for Health Analytic Research, LLC and were paid by Ipsen Biopharmaceuticals to conduct this study. SJP, BM, and DC are employees of Ipsen Biopharmaceuticals. ATP serves as a consultant for Ipsen Biopharmaceuticals. Since completion of this work, ATP's affiliation has changed to Morehouse College and Georgia Institute of Technology. The authors report no other conflicts of interests in this work.

\section{References}

1. Pearse AG. The cytochemistry and ultrastructure of polypeptide hormone-producing cells of the APUD series and the embryologic, physiologic and pathologic implications of the concept. J Histochem Cytochem. 1969;17(5):303-313.

2. Moertel CG. Karnofsky memorial lecture. An odyssey in the land of small tumors. J Clin Oncol. 1987;5(10):1502-1522.

3. Yao JC, Hassan M, Phan A, et al. One hundred years after "carcinoid": epidemiology of and prognostic factors for neuroendocrine tumors in 35,825 cases in the United States. J Clin Oncol. 2008;26(18):3063-3072.

4. Halfdanarson TR, Rabe KG, Rubin J, Petersen GM. Pancreatic neuroendocrine tumors (PNETs): incidence, prognosis and recent trend toward improved survival. Ann Oncol. 2008;19(10):1727-1733.

5. Berardi R, Rinaldi S, Torniai M, et al. Gastrointestinal neuroendocrine tumors: searching the optimal treatment strategy. A literature review. Crit Rev Oncol Hematol. 2016;98:264-274.

6. Rinke A, Müller HH, Schade-Brittinger C, et al. Placebo-controlled, double-blind, prospective, randomized study on the effect of octreotide LAR in the control of tumor growth in patients with metastatic neuroendocrine midgut tumors: a report from the PROMID Study Group. J Clin Oncol. 2009;27(28):4656-4663.

7. Caplin ME, Pavel M, Ćwikła JB, et al. Lanreotide in metastatic enteropancreatic neuroendocrine tumors. NEngl JMed. 2014;371(3):224-233.

8. Caplin ME, Pavel M, Ćwikła JB, et al. Anti-tumour effects of lanreotide for pancreatic and intestinal neuroendocrine tumours: the CLARINET open-label extension study. Endocr Relat Cancer. 2016;23(3):191-199.

9. Rinke A, Wittenberg M, Schade-Brittinger C, et al. Placebo-controlled, double-blind, prospective, randomized study on the effect of octreotide LAR in the control of tumor growth in patients with metastatic neuroendocrine midgut tumors (PROMID): results of long-term survival. Neuroendocrinology. 2017;104(1):26-32.

10. National Comprehensive Cancer Network, Inc. NCCN Clinical Practice Guidelines in Oncology (NCCN Guidelines $\left.{ }^{\circledR}\right)$ : Neuroendocrine Tumors Version 1.2015. November 2014.

11. NCCN Guidelines ${ }^{\circledR} \&$ Clinical Resources. NCCN Drugs \& Biologics Compendium $^{\circledR}$. Available from: https://www.nccn.org/professionals/ drug_compendium/content/contents.asp. Accessed April 24, 2015.

12. Financial Burden of Cancer Care|Cancer Trends Progress Report. Available from: http://progressreport.cancer.gov/after/economic_ burden\#field_most_recent_estimates. Accessed May 3, 2016.

13. Epstein AM, Jha AK, Orav EJ, et al. Analysis of early accountable care organizations defines patient, structural, cost, and quality-of-care characteristics. Health Aff (Millwood). 2014;33(1):95-102.

14. Peppercorn J. The financial burden of cancer care: do patients in the US know what to expect?. Expert Rev Pharmacoecon Outcomes Res. 2014;14(6):835-842.

15. Mauskopf JA, Sullivan SD, Annemans L, et al. Principles of good practice for budget impact analysis: report of the ISPOR task force on good research practices budget impact analysis. Value Health. 2007;10(5):336-347.

16. Sullivan SD, Mauskopf JA, Augustovski F, et al. Budget impact analysisprinciples of good practice: report of the ISPOR 2012 budget impact analysis good practice II task force. Value Health. 2014;17(1):5-14.
17. Modlin IM, Oberg K, Chung DC, et al. Gastroenteropancreatic neuroendocrine tumours. Lancet Oncol. 2008;9(1):61-72.

18. Ferone D, Forsythe A, Bakker B, Pedersini R, Goren A. Real world data on patients with neuroendocrine tumors treated with octreotide LAR. Poster presented at: European Cancer Congress 2013 (ESMO); September 27 - October 1 2013; Amsterdam, Netherlands.

19. Hallet J, Law CH, Cukier M, Saskin R, Liu N, Singh S. Exploring the rising incidence of neuroendocrine tumors: a population-based analysis of epidemiology, metastatic presentation, and outcomes. Cancer. 2015;121(4)589-597.

20. Pokuri VK, Fong MK, Iyer R. Octreotide and lanreotide in gastroenteropancreatic neuroendocrine tumors. Curr Oncol Rep. 2016;18(1):7.

21. Strosberg J, Casciano R, Stern L, et al. United States-based practice patterns and resource utilization in advanced neuroendocrine tumor treatment. World J Gastroenterol. 2013;19(15):2348-2354.

22. Wolters Kluwer. Price Rx. Available from: https://pricerx.medispan. com/. Published 2016. Accessed April 25, 2017.

23. Novartis Pharmaceuticals Corporation. Sandostatin LAR [Prescribing Information]. December 2014. Available from: http://www.pharma. us.novartis.com/product/pi/pdf/sandostatin_lar.pdf. Accessed April 20, 2017.

24. SOMATULINE ${ }^{\circledR}$ DEPOT (lanreotide) injection [prescribing Information]. Ipsen Biopharmaceuticals, Inc; 2014.

25. Strosberg JR, Benson AB, Huynh L, et al. Clinical benefits of abovestandard dose of octreotide LAR in patients with neuroendocrine tumors for control of carcinoid syndrome symptoms: a multicenter retrospective chart review study. The Oncologist. 2014;19(9):930-936.

26. Xu Y, Shih YT, Leary C, Shen C, Yao JC. Dosing patterns of octreotide LAR among elderly patients with neuroendocrine tumors: Analysis of the SEER-Medicare database. J Clin Oncol. 2012;30(Suppl):e14550.

27. Shen $\mathrm{C}, \mathrm{Xu}$ Y, Yao JC, Shih YT. Octreotide LAR use among elderly patients with carcinoid syndrome and survival outcomes: a populationbased analysis. J Clin Oncol. 2012;30(Suppl 4):322.

28. Kulke MH, Horsch D, Caplin M, et al. 37LBA Telotristat etiprate is effective in treating patients with carcinoid syndrome that is inadequately controlled by somatostatin analog therapy (the phase 3 TELESTAR clinical trial). Eur J Cancer. 2015;51(Suppl 3):S728.

29. Maxine D. Fisher, Sonia Pulgar, et al. Treatment patterns and outcomes in metastatic neuroendocrine tumors: results from a retrospective community oncology database. Presented at: North American Neuroendocrine Tumor Society Symposium; 15-17 October, 2015; Austin, TX, USA.

30. Jonathan R Strosberg, Sarah Bobiak, Carrie C Zornosa, et al. Dosing patterns for octreotide LAR in neuroendocrine tumor (NET) patients: NCCN NET outcomes database. J Clin Oncol. 2013;31(Suppl 15): 4142.

31. U.S. Bureau of Labor Statistics. Occupation Employment Statistics. Available from: http://www.bls.gov/oes/current/oes291171.htm. Published March 2016. Accessed March 28, 2016.

32. Adelman DT, Burgess A, Davies PR. Evaluation of long-acting somatostatin analog injection devices by nurses: a quantitative study. Med Devices (Auckl). 2012;5:103-109.

33. Boyd AE, DeFord LL, Mares JE, et al. Improving the success rate of gluteal intramuscular injections. Pancreas. 2013;42(5):878-882.

34. Zaybak A, Güneş ÜY, Tamsel S, Khorshid L, Eser I. Does obesity prevent the needle from reaching muscle in intramuscular injections?. J Adv Nurs. 2007;58(6):552-556.

35. Cockshott WP, Thompson GT, Howlett LJ, Seeley ET. Intramuscular or intralipomatous injections?. N Engl J Med. 1982;307(6):356-358.

36. Nisbet AC. Intramuscular gluteal injections in the increasingly obese population: retrospective study. BMJ. 2006;332(7542):637-638.

37. Chan VO, Colville J, Persaud T, Buckley O, Hamilton S, Torreggiani WC. Intramuscular injections into the buttocks: are they truly intramuscular? Eur J Radiol. 2006;58(3):480-484.

38. Fazio N, Spada F. Real-word analysis on the use of somatostatin analogs (SSAs) in patients with neuroendocrine neoplasms (NENs): results of a survey conducted by the Italian Society of Medical Oncology (AIOM). Ann Oncol. 2016;27(suppl 4):iv114. 
39. Modica R, Ramundo V, Marciello F, et al. High-dose treatment with somatostatin analogues in neuroendocrine tumours. Endocr Abstr. 2015;37:EP1132.

40. Ruszniewski P, Valle JW, Lombard-Bohas C, et al. Patient-reported outcomes with lanreotide Autogel/Depot for carcinoid syndrome: an international observational study. Dig Liver Dis. 2016;48(5): $552-558$.

41. Van Fraeyenhove F, Meireson N, Terriere L, et al. High-dose lanreotide in the treatment of poorly differentiated pancreatic neuroendocrine carcinoma: a case report. Case Rep Oncol. 2014;7(1):155-163.

42. Strosberg J, El-Haddad G, Wolin E, et al. Phase 3 trial of ${ }^{177} \mathrm{Lu}-$ Dotatate for midgut neuroendocrine tumors. N Engl J Med. 2017;376: 125-135.
43. Novartis Pharmaceuticals Corporate Fact Sheet. January 2016. Available from: https://www.novartis.com/sites/www.novartis.com/files/2a_Pharmaceuticals_EN.pdf. Accessed May 4, 2016.

44. Rajeev Ayyagari, Maureen Neary, Shang Li, et al. Economic evaluation of octreotide LAR versus lanreotide depot in the treatment of metastatic gastrointestinal neuroendocrine tumors. Presented at: North American Neuroendocrine Tumor Society Symposium; 10-11 October, 2014; Nashville, TN, USA.

45. Vinik Aaron, Wolin Edward M, Adury Helene, Gomez-Panzani Edda L. ELECT: A phase 3 study of efficacy and safety of lanreotide autogel/depot (LAN) treatment for carcinoid syndrome in patients with neuroendocrine tumors (NETs) [abstract]. J Clin Oncol. 2014. Available from: http:// meetinglibrary.asco.org/content/123139-143. Accessed July 22, 2016.
ClinicoEconomics and Outcomes Research

\section{Publish your work in this journal}

ClinicoEconomics and Outcomes Research is an international, peerreviewed open-access journal focusing on health technology assessment, pharmacoeconomics and outcomes research in the areas of diagnosis, medical devices, and clinical, surgical and pharmacological intervention. The economic impact of health policy and health systems
Submit your manuscript here: https://www.dovepress.com/clinicoeconomics-and-outcomes-research-journal

\section{Dovepress}

organization also constitute important areas of coverage. The manuscript management system is completely online and includes a very quick and fair peer-review system, which is all easy to use. Visit http://www.dovepress.com/testimonials.php to read real quotes from published authors. 